\title{
THE VALUE OF $J$ AT A SAMELSON PRODUCT ${ }^{1}$
}

\section{GEORGE MCCARTY}

Introduction. At the Seattle Conference, 1963, M. G. Barratt asked the question, "Can anything general be said about $J: \pi_{k}\left(S O_{n}\right)$ $\rightarrow \pi_{k+n}\left(S^{n}\right)$ on Samelson products?" Lashof records [4] that Stasheff pointed out an answer to this question on the basis of results obtained by B. Steer in his Oxford thesis [8]. We offer here another answer, phrased in quite different terms, which is simple in both statement and proof.

Let $p: O_{n+1} \rightarrow S^{n}$ be the usual bundle map with fiber $O_{n}$; in [5] we define the " $p$-product": $\pi_{q}\left(O_{n}\right) \otimes \pi_{s}\left(S^{n}\right) \rightarrow \pi_{q+s}\left(S^{n}\right)$. The $p$-product of $\alpha \in \pi_{q}\left(O_{n}\right)$ with $\beta \in \pi_{s}\left(S^{n}\right)$ is denoted $[\alpha, \beta]_{p}$. This product generalizes the $J$ homomorphism: if $\iota_{n}$ denotes the class of the identity map on $S^{n}$ then $J(\alpha)=\left[\alpha, \iota_{n}\right]_{p}[\mathbf{5}$, Corollary 5.7].

Theorem. Let $\alpha \in \pi_{q}\left(O_{n}\right), \alpha^{\prime} \in \pi_{q^{\prime}}\left(O_{n}\right)$, and let $\left\langle\alpha, \alpha^{\prime}\right\rangle$ denote their Samelson product (see [3]). Then

$$
J\left\langle\alpha, \alpha^{\prime}\right\rangle=\left[\alpha, J \alpha^{\prime}\right]_{p}-(-1)^{q q^{\prime}}\left[\alpha^{\prime}, J \alpha\right]_{p} .
$$

The p-product is defined in terms of the "mixed product":

$$
\pi_{q}\left(O_{n}\right) \otimes \pi_{s}\left(O_{n+1}, O_{n}\right) \rightarrow \pi_{q+s}\left(O_{n+1}, O_{n}\right) ;
$$

the product of $\alpha \in \pi_{q}\left(O_{n}\right)$ with $\gamma \in \pi_{s}\left(O_{n+1}, O_{n}\right)$ is denoted $\langle\alpha, \gamma\rangle$, and $[\alpha, \beta]_{p}=(-1)^{a} p_{*}\left\langle\alpha, p_{*}{ }^{-1} \beta\right\rangle$ by definition [5]. Hence our theorem may be restated as

$$
p_{*}^{-1} J\left\langle\alpha, \alpha^{\prime}\right\rangle=(-1)^{q}\left\langle\alpha, p_{*}^{-1} J \alpha^{\prime}\right\rangle-(-1)^{q^{\prime} q+q^{\prime}}\left\langle\alpha^{\prime}, p_{*}^{-1} J \alpha\right\rangle .
$$

Since $J$ may be expressed as a mixed product, this latter statement may readily be translated in to a Jacobi identity. We prove the theorem by establishing, for group pairs $(G, H)$ (where $H$ is a subgroup of $G$ ), an identity for the mixed product $\pi_{q}(H) \otimes \pi_{s}(G, H) \rightarrow \pi_{q+s}(G, H)$ :

$$
(-1)^{q s}\left\langle\left\langle\alpha, \alpha^{\prime}\right\rangle, \gamma\right\rangle+(-1)^{q^{\prime} s}\left\langle\langle\gamma, \alpha\rangle, \alpha^{\prime}\right\rangle+(-1)^{q q^{\prime}}\left\langle\left\langle\alpha^{\prime}, \gamma\right\rangle, \alpha\right\rangle=0 .
$$

This follows from two lemmas, each of some independent interest. (Each loop space below is meant to be of the type introduced by Moore.)

Lemma 1. The Jacobi identity holds for the $H$-pair $(\Omega Y, \Omega B)$ of loop spaces of a pair $(Y, B)$ of spaces.

Received by the editors December 6, 1966.

${ }^{1}$ I am grateful to NSF for the support of this work through their grant GP 5288. 
Lemma 2. For each group pair $(G, H)$ which satisfies the first axiom of countability there exists a classifying pair $\left(B_{G}, B_{H}\right)$ and a weak homotopy equivalence $f:(G, H) \rightarrow\left(\Omega B_{G}, \Omega B_{H}\right)$ which preserves mixed products.

(We certainly would expect a stronger form of this lemma to hold.)

The author has appreciated stimulating conversations on this question with Eldon Dyer and Richard Lashof.

Proof of Lemma 1. We introduce a method of "universal examples" for $H$-constructions which parallels a device of Blakers and Massey [1]. Let $B$ be a space (with basepoint), $\partial: \pi_{q+1}(B) \cong \pi_{q}(\Omega B)$ and let $a: S^{q+1} \rightarrow B$ represent $\partial^{-1}(\alpha)$ for $\alpha \in \pi_{q}(\Omega B)$. Then $a$ induces an exact $H$-map $\bar{a}: \Omega S^{q+1} \rightarrow \Omega B$ defined by $\bar{a}(l)=a \circ l$. Further, if $\iota_{q+1}$ is the class of the identity map on $S^{q+1}$ and $\bar{\imath}_{q}=\partial\left(\iota_{q+1}\right)$, then $\bar{a}_{*}\left(\bar{\iota}_{q}\right)=\alpha$. A modification of the above for a pair $(Y, B)$ uses $\partial: \pi_{s+1}(Y, B) \cong \pi_{s}(\Omega Y, \Omega B)$ to define from $\gamma \in \pi_{s}(\Omega Y, \Omega B)$ a map $c:\left(I^{s+1}, S^{s}\right) \rightarrow(Y, B)$ and, in turn, an exact $H$-map $\bar{c}:\left(\Omega I^{s+1}, \Omega S^{s}\right) \rightarrow(\Omega Y, \Omega B)$. If $\kappa_{s+1}$ is the class of the identity on $\left(I^{s+1}, S^{s}\right)$ and $\bar{\kappa}_{s}=\partial\left(\kappa_{s+1}\right) \in \pi_{s}\left(\Omega I^{s+1}, \Omega S^{s}\right)$ then $\bar{c}_{*}\left(\bar{\kappa}_{s}\right)=\gamma$. Again, if $\alpha$ and $\gamma$ together define a map from $\left(S^{q+1} \vee I^{s+1}, S^{q+1} \vee S^{s}\right)$ into $(Y, B)$ and thus an exact $H$-map $g$ : [ $\left.\Omega\left(S^{q+1} \bigvee I^{s+1}\right), \Omega\left(S^{q+1} \bigvee S^{s}\right)\right]$ $\rightarrow(Y, B)$, and if $\bar{i}_{q}$ and $\bar{\kappa}_{s}$ denote their own images under appropriate inclusions into $\Omega\left(S^{q+1} \bigvee S^{s}\right)$ and $\left[\Omega\left(S^{q+1} \bigvee I^{s+1}\right), \Omega\left(S^{q+1} \vee S^{s}\right)\right]$, then the naturality of the mixed product implies

$$
g_{*}\left\langle\bar{\imath}_{q}, \bar{\kappa}_{s}\right\rangle=\left\langle g_{*} \bar{i}_{q}, g_{*} \bar{\kappa}_{s}\right\rangle=\langle\alpha, \gamma\rangle .
$$

Yet another such construction uses representatives of $\alpha \in \pi_{q}(\Omega B)$, $\alpha^{\prime} \in \pi_{q^{\prime}}(\Omega B)$ and $\gamma \in \pi_{s}(\Omega Y, \Omega B)$ to construct an exact $H$-map

$$
h:\left[\Omega\left(S^{q+1} \vee S^{q^{\prime}+1} \vee I^{s+1}\right), \Omega\left(S^{q+1} \vee S^{q^{\prime+1}} \vee S^{s}\right)\right] \rightarrow(\Omega Y, \Omega B)
$$

with the property that $h_{*}\left\langle\left\langle\bar{\iota}_{q}, \bar{\iota}_{q^{\prime}}\right\rangle, \bar{\kappa}_{8}\right\rangle=\left\langle\left\langle\alpha, \alpha^{\prime}\right\rangle, \gamma\right\rangle$, etc. Now observe that in the homotopy sequence

$$
\begin{aligned}
& \cdots \rightarrow \pi_{n}\left[\Omega\left(S^{q+1} \vee S^{q^{\prime}+1} \vee I^{s+1}\right), \Omega\left(S^{q+1} \vee S^{q^{\prime}+1} \vee S^{s}\right) ।\right. \\
& \quad \stackrel{\partial}{\rightarrow} \pi_{n-1}\left[\Omega\left(S^{q+1} \vee S^{q^{\prime}+1} \bigvee S^{s}\right)\right] \\
& \quad \stackrel{\pi_{n-1}}{\rightarrow}\left[\Omega\left(S^{q+1} \vee S^{q^{\prime}+1} \bigvee I^{s+1}\right)\right] \rightarrow \cdots
\end{aligned}
$$

$i$ is an epimorphism in all dimensions, so that $\partial$ is a monomorphism. But the relations $\partial\langle\alpha, \gamma\rangle=\langle\alpha, \partial \gamma\rangle$ and $\partial\langle\gamma, \alpha\rangle=(-1)^{q}\langle\partial \gamma, \alpha\rangle$ between the mixed products in the domain of $\partial$ and the absolute (Samelson) products in the range carry a "Jacobi sum" in the domain to a Jacobi sum in the range. Since the Jacobi relation holds for the absolute product [3], it must hold in the domain of $\partial$. It is now clear that if the 
morphism $h_{*}$ is defined by $\alpha, \alpha^{\prime}$ and $\gamma$ then

$$
\begin{aligned}
h_{*}\left((-1)^{q g}\left\langle\left\langle\bar{i}_{q}, i_{q^{\prime}}\right\rangle, \bar{\kappa}_{s}\right\rangle+(-1)^{s q^{\prime}}\left\langle\left\langle\bar{\kappa}_{8}, \bar{\imath}_{q}\right\rangle, i_{q^{\prime}}\right\rangle+(-1)^{q^{\prime} q}\left\langle\left\langle\bar{i}_{q^{\prime}}, \bar{\kappa}_{8}\right\rangle, \bar{i}_{q}\right\rangle\right) \\
=(-1)^{q s}\left\langle\left\langle\alpha, \alpha^{\prime}\right\rangle, \gamma\right\rangle+(-1)^{s q^{\prime}}\left\langle\langle\gamma, \alpha\rangle, \alpha^{\prime}\right\rangle+(-1)^{q^{\prime} q}\left\langle\left\langle\alpha^{\prime}, \gamma\right\rangle, \alpha\right\rangle \\
=0 \in \pi_{q+q^{\prime}+s}(\Omega Y, \Omega B) .
\end{aligned}
$$

Proof of Lemma 2. Let $(G, H)$ be a group pair; Milnor [6] gives a construction of a universal bundle $E_{G}$ with base space $B_{G}$ and fiber $G$. Examination of this construction shows that the corresponding universal bundle $E_{H} \rightarrow B_{H}$ with fiber $H$ is a subbundle of $E_{G} \rightarrow B_{G}$; that is, $(G, H) \rightarrow\left(E_{G}, E_{H}\right) \rightarrow\left(B_{G}, B_{H}\right)$ is a fibering of pairs. An explicit recipe for a contraction of $E_{G}$ is offered by Dold [2]; his function moves $E_{H}$ through $E_{H}$ to a point, and so is a contraction of the pair $\left(E_{G}, E_{H}\right)$. Samelson [7] describes an $H$-map from $G$ to the usual loop space of $B_{G}$ which is a weak homotopy equivalence; his map may be constructed from a given contraction $k$ of $E_{G}$ and it thus defines a map of the pair $(G, H)$ into the pair of usual loop spaces of $B_{G}$ and $B_{I I}$. This pair map is a weak homotopy equivalence, by the five lemma.

We now alter Samelson's map to define a weak homotopy equivalence from $(G, H)$ into the pair $\left(\Omega B_{G}, \Omega B_{H}\right)$ of Moore-loop spaces. Let $k: E_{G} \times I \rightarrow E_{G}$ be the contraction of $E_{G}$ to $e \in G$ described by Dold, and let $f$ be a nonnegative real valued function on $G$ with $f^{-1}(0)=e$ (the existence of such an $f$ is easily shown for first countable groups). For each $x \in G$ define $\phi(x) \in \Omega B_{G}$ to be the loop $\phi(x):[0, f(x)]$ $\rightarrow B_{G}$ whose values are, for $x \neq e$,

$$
\phi(x)(u)=p \circ k[x, u / f(x)] .
$$

(Here $p:\left(E_{G}, E_{H}\right) \rightarrow\left(B_{G}, B_{H}\right)$ is the bundle projection.) Since $\phi$ is clearly homotopic to the map defined by Samelson when the latter is regarded as having range $\Omega B_{G}, \phi$ is a weak homotopy equivalence, and $\phi(H) \subset \Omega B_{H}$.

Next we define a homotopy $\mathcal{F}: G \times G \times I \rightarrow \Omega B_{G}$ which shows $\phi$ to be a strong $H$-map (that is, $\mathfrak{H C}(x, e, t)=\mathfrak{H}(e, x, t)$ for all $t)$ of pairs.

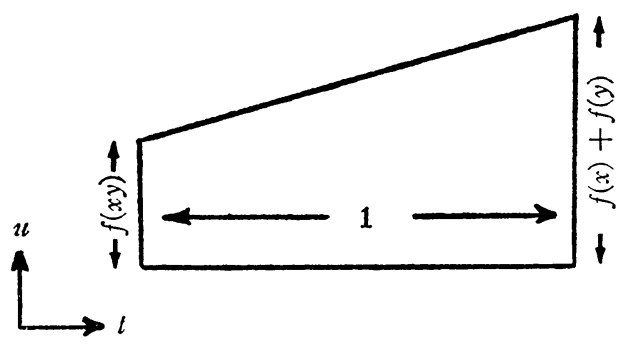


The existence of $\mathscr{H C}$ implies that $\phi$ preserves mixed products $[5, \mathrm{Sec}-$ tion 4]. For simplicity of notation, we identify $(G, H)$ with the distinguished fiber of $\left(E_{G}, E_{H}\right)$ and denote the right action of $(G, H)$ on $\left(E_{G}, E_{H}\right)$ by juxtaposition; $e$ is the identity of $G$. For each $(x, y)$

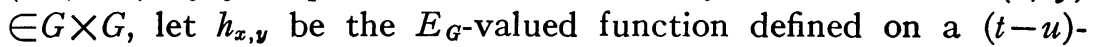
trapezoid as follows: on the bottom edge $h_{x, y}(t, 0)=x y$, on the left edge $h_{x, y}(0, u)=k[x y, u / f(x y)]$, on the top edge $h_{x, y}(t, u)=e$, and along the right edge

$$
\begin{aligned}
h_{x, \nu}(1, u) & =k[x, u / f(x)] y & & \text { if } 0 \leqq u \leqq f(t), \\
& =k[y,(u-f(x)) / f(y)] & & \text { if } f(x) \leqq u \leqq f(x)+f(y) .
\end{aligned}
$$

Now let $h_{x, y}$ be defined at the center of the trapezoid by

$$
h_{x, y}\left(\frac{1}{2}, \frac{f(x y)+f(x)+f(y)}{2}\right)=e
$$

and extend $h_{x, y}$ to the whole figure by letting the contraction $k$ act in the obvious linear fashion along each ray from center to boundary. The only degenerate case is $x=y=e$; there $h_{e, e}=e$. Let $p$ be the projection of the bundle $\left(E_{G}, E_{H}\right)$ and define $\mathfrak{H C}(x, y, t)(u)=p \circ h_{x, y}(t, u)$; since the right action of $G$ on $E_{G}$ has trivial projection, each value of $\mathfrak{H C}$ is clearly a Moore loop in $B_{G}$. Further, $\mathcal{H}(x, y, 0)=\phi(x, y)$, $\mathfrak{H}(x, y, 1)=\phi(x) \phi(y)$, and $\mathfrak{H}(x, e, t)=\mathfrak{F}(e, x, t)$ for all $x$ and $t$; hence $\phi$ is a strong $H$-map.

\section{REFERENCES}

1. A. L. Blakers and W. S. Massey, Products in homotopy theory, Ann. of Math. 58 (1953), 295-324.

2. A. Dold, Partitions of unity in the theory of fibrations, Ann. of Math. 78 (1963), 223-255; p. 251.

3. I. M. James, On H-spaces and their homotopy groups, Quart. J. Math. Oxford Ser. (2) 11 (1960), 161-179; p. 170.

4. R. Lashof, Editor, Problems in differential and algebraic topology, Seattle Conference, 1963, Ann. of Math. 81 (1965), 565-591; p. 588.

5. G. McCarty, Products between homotopy groups and the J-morphism, Quart. J. Math. Oxford Ser. (2) 15 (1964), 362-370. 436.

6. J. Milnor, Construction of universal bundles. II, Ann. of Math. 63 (1956), 430-

7. H. Samelson, Groups and spaces of loops, Comment. Math. Helv. 28 (1954), 278-287.

8. B. Steer, Extensions of mappings into $H$-spaces, Proc. London Math. Soc. (3) 13 (1963), 219-272; Theorem 5.76.

University of California, Irvine 